\title{
Study of the effect of transport current and combined transverse and longitudinal fields on the ac loss in NET prototype conductors
}

\author{
A. Nijhuis and H. H. J. ten Kate \\ Applied Superconductivity Centre, University of Twente \\ P.O. Box 217, 7500 AE Enschede, The Netherlands.
}

\begin{abstract}
AC losses in cables carrying dc as well as ac transport currents at different dc background fields up to $2 \mathrm{~T}$ have been measured on three types of $\mathrm{Nb}_{3} \mathrm{Sn}$ subcables in a new test facility. In this facility it is possible to apply sinusoidal transverse ac fields up to $\mathrm{dB} / \mathrm{dt}=\mathrm{5T} / \mathrm{s}$ and longitudinal ac fields up to $d B / d t=30 T / s$ separately and simultaneously. The ac loss is measured with a calorimetric method. Simultaneously applied transverse and longitudinal fields can result in a loss which exceeds the added contributions of the separate applied ac fields. Within the measured range it is about correct (within $10 \%$ ) to add the loss components due to dc transport current up to $10 \mathrm{kA}$ and both applied transverse and longitudinal ac fields. The measured total loss is always above the sum of the loss components.
\end{abstract}

\section{INTRODUCTION.}

As part of the NET conductor development program a study of ac loss in 3 types of experimental sub cables is performed in order to investigate the effects of the type of cabling , and the application of transverse and longitudinal fields and transport current at the same time.

The first loss measurements with the new test facility built in Twente [1] were performed with transverse and longitudinal bipolar sine wave ac fields with respect to the axis of the cable, at different frequencies, without transport current and with different dc background fields.

From these results the hysteresis and coupling losses can be determined assuming that the hysteresis loss per cycle is independent from the frequency (which is true for low frequencies and when no internal shielding is present). Then both components can be separated using $Q_{\text {tot }}=Q_{\text {hys }}+$ $Q_{c p l}$, where $Q$ is the loss per cycle and per unit conductor volume. This can, at a constant amplitude of the ac field, be written as $Q_{\text {tot }}=c+k . f$, where $f$ is the frequency and $c$ and $k$ are constants. The results of these experiments are published elsewhere [2,3].

In addition a current can be introduced in the cable (using the superconducting transformer) in order to investigate the

Manuscript received September 20 1993. These investigations are carried out as part of the contract NET/91-277 between the NET-team in Garching and the University of Twente. influences of dc as well as ac transport currents on the cable loss. The main part of the results of the loss measurements with dc transport current is reported in other articles $[3,4]$.

The last part of the measurement programme and main subject of this paper is the study of the interaction of simultaneously applied transverse and longitudinal ac fields in combination with ac and dc transport currents and a dc background field.

\section{LOSS DUE TO TRAN̈SVERSE AND LONGITUDINAL AC FIELDS (NO TRANSPORT CURRENT).}

From the results of all introducing measurements without transport current only the main results are summarised.

In Table I the specifications of the three sub cables subjected to the experiments are given [5]. The $\mathrm{Nb}_{3} \mathrm{Sn}$ strand material is manufactured by TWCA and the internal structure is of the MJR (modified jelly roll) type.

TABLE I

SPBCIFICATTONS OF THB SUB CABLES USED IN THE EXPERRMENTS

\begin{tabular}{lcccccc}
\hline Cable \# & Type & $\begin{array}{c}\text { No. of } \\
\text { strands }\end{array}$ & $\begin{array}{c}\varnothing_{\text {strmid }} \\
{[\mathrm{mm}]}\end{array}$ & $\begin{array}{c}\text { No. of } \\
\text { MJRs }\end{array}$ & $\begin{array}{c}\sigma_{\text {MIR }} \\
{[\mu \mathrm{m}]}\end{array}$ & Cabled by \\
\hline \#B1 & Braided & 29 & 0.93 & 54 & 44 & ABB \\
\#T1 & Twisted & 27 & 0.96 & 54 & 44 & ABB \\
\#T2 & Twisted & 27 & 0.78 & 18 & 60 & LMI \\
\hline
\end{tabular}

The hysteresis loss per cycle per conductor volume, $Q_{\text {hys, }}$ can be described as linear for $\mathrm{B}_{\mathrm{a}}>\mathrm{B}_{\mathrm{p}}: \mathrm{Q}_{\mathrm{hys}}=\mathrm{q} .\left(\mathrm{B}_{\mathrm{a}}-\mathrm{b}\right)$ in which $q$ and $b$ are constants and $B_{a}$ is the amplitude of the applied ac field. For cable \#B1 the hysteresis loss with $\mathrm{B}_{\mathrm{dc}}=2 \mathrm{~T}$ can be written as $\mathrm{Q}_{\text {hys }}=274 .\left(\mathrm{B}_{\mathrm{a}}-0.198\right)$ $\mathrm{mJ} / \mathrm{cm}^{3}$.cycle. The main results of the measurements of the hysteresis loss for tránsverse and longitudinal fields in a background field $B_{\mathrm{dc}}=2 \mathrm{~T}$ are summarised in Table II.

There is no basic difference between the B1- and T1-type of cables concerning the behaviour of the hysteresis loss. The strand used for cable \#T2 shows noticeable lower hysteresis loss for transverse fields. This result is opposite with respect to the case of longitudinal ac field and can be due to the internal geometry of the strand material. The layout of the MJR subelements is the same and an identical critical current density is measured [5]. Also the $\mathrm{Cu} / \mathrm{nonCu}$ 
ratio for both strands is 1.25 . The number and the size of the MJR's assembled in the two strands is different which can cause a difference in the effective filament diameter. For this reason it is expected that cable \#T2 would show higher hysteresis loss with respect to cables \#T1 and \#B1. No explanation is found for this.

TABLE II

COMPARISON OF THE HYSTERESIS LOSS FOR TRANSVERSE AND LONGTUDNAL AC FIEIDS AT $B_{D C}=2 T(B A>B P)$.

\begin{tabular}{|c|c|c|c|}
\hline $\mathrm{B}_{\mathrm{a}}$ & $\# \mathrm{~B} 1$ & \#T1 & \#T2 \\
\hline trans & $q \cdot\left(B_{a}-b\right)$ & $0.97 . q \cdot\left(B_{a}-1.03 . b\right)$ & $0.68 . \mathrm{q} \cdot(\mathrm{B},-0.98 . \mathrm{b})$ \\
\hline long & $0.055 . q .\left(B_{a}-0.31 . b\right)$ & $0.055 . q .\left(B_{2}-0.31 . b\right)$ & $0.062 . q .\left(B_{-}-0.32 . b\right)$ \\
\hline
\end{tabular}

The coupling loss, as a first and simple approximation, is taken proportional to the frequency $f$ and to $B_{a}{ }^{2}$. This is usually formulated as:

$$
\mathrm{Q}_{\mathrm{cpl}}=\left(2 \pi / \mu_{0}\right) \cdot \mathrm{B}_{\mathrm{a}}{ }^{2} \cdot \omega \cdot \tau \quad\left[\mathrm{J} /\left(\mathrm{m}^{3} \cdot \mathrm{cycle}\right)\right] .
$$

The time constant is $\tau=1 / 2 \cdot \mu_{0} \cdot\left(\mathrm{L}_{\mathrm{p}} / 2 \pi\right)^{2} \cdot \sigma_{\perp}$ [s] in which $\mathrm{L}_{\mathrm{p}}$ is the twist pitch, and $\sigma_{\perp}$ is the effective electrical conductivity in the transverse direction. In table III a comparison of the coupling loss time constants of the three cables as determined calorimetrically for both directions of the ac field is shown. The coupling loss time constant found for cable $\# \mathrm{Bl}$ in a background field $\mathrm{Bdc}=2 \mathrm{~T}$ amounts to $\tau$ $=0.60 \mathrm{~ms}$.

TABLB III

COMPARISON OF THE COUPLING LOSS TIMB CONSTANTS P FOUND FOR TRANSVERSE AND LONGITUDINAL APPLIED AC FIELDS WTTH $B_{D C}=2 T$.

\begin{tabular}{cccc}
\hline & $\# \mathrm{~B} 1$ & $\# \mathrm{~T} 1$ & $\# \mathrm{~T} 2$ \\
\hline $\mathrm{Ba}$ (trans) & $\tau$ & $0.7 \tau$ & $0.6 \tau$ \\
$\mathrm{Ba}$ (long) & $0.023 \tau$ & $0.058 \tau$ & $0.031 \tau$ \\
\hline
\end{tabular}

In the case of a transverse ac field the losses of the braided cable are higher than for both twisted types. For parallel ac fields this relation is opposite.

\section{THE INFLUENCE OF TRANSPORT CURRENT ON THE LOSS.}

\section{A. DC transport current.}

The measured maximum current is about $50 \%$ of the estimated critical current in all cables. This reduced performance can be caused by unstable flux jump behaviour in combination with an increased sensitivity for quenches at low background fields due to the large effective filament diameter and the weak mechanical suspension of the strands in these non jacketed cables. For this reason it was not possible to investigate the behaviour near to the critical current of the cables, which are approximately $43 \mathrm{kA}$ for \#B1 and \#T1 and $17 \mathrm{kA}$ for \#T2 respectively.

No relation between the maximum transport current and the ramp rate is observed in the range $0.1<\mathrm{dI}_{\mathrm{T}} / \mathrm{dt}<4 \mathrm{kA} / \mathrm{s}$ where the quench current is on a plateau and at about $50 \%$ of the critical current. All cables show the same unstable behaviour for $\mathrm{dc}$ transport currents exceeding $60 \%$ of the quench current. It is sure that the low quench current is not caused by heat development in the joints. The joint resistance is measured and agrees with the design value of $\mathrm{R}_{\text {joint }}=1 \mathrm{n} \Omega$.

The contribution of the transport current to the loss partly consists of interstrand coupling loss which change with the interstrand resistance which is influenced by the Lorentz forces on the strands in the cable. A comparison of the influence of the transport current on the total loss between different transverse and longitudinal ac field amplitudes is described elsewhere [4].

\section{B. AC transport current.}

In order to measure the additional loss caused by an ac transport current it is necessary to superpose the triangular ac current on the dc current which is equal or higher in magnitude. The Lorentz forces on the cable due to the transverse dc field must be directed towards the centre of the sample holder [1] to prevent the cable from damage and getting loose from the groove in the sample holder. The $d c$ transport current in the test is $5 \mathrm{kA}$ and $\mathrm{B}_{\mathrm{de}}$ is $2 \mathrm{~T}$.

The relation between the loss and the amplitude of the triangular superposed ac current in Figure 1, starts with the third power of transport current and will probably become linear at higher amplitudes.

The total loss generated in the cable consists of transportcurrent loss, shielding loss and coupling loss. The loss for different ramprates of current coincide, which

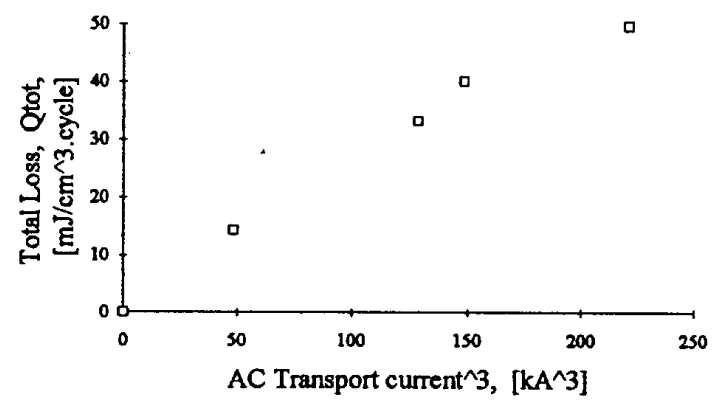

Fig $1 Q_{\text {tot }}=\mathrm{fI}_{\mathrm{T}^{3 c}}$, without transverse ac field, $\mathrm{I}_{\mathrm{Tdc}}=5 \mathrm{kA}, \mathrm{B}_{\mathrm{dc}}=2 \mathrm{~T}$. 


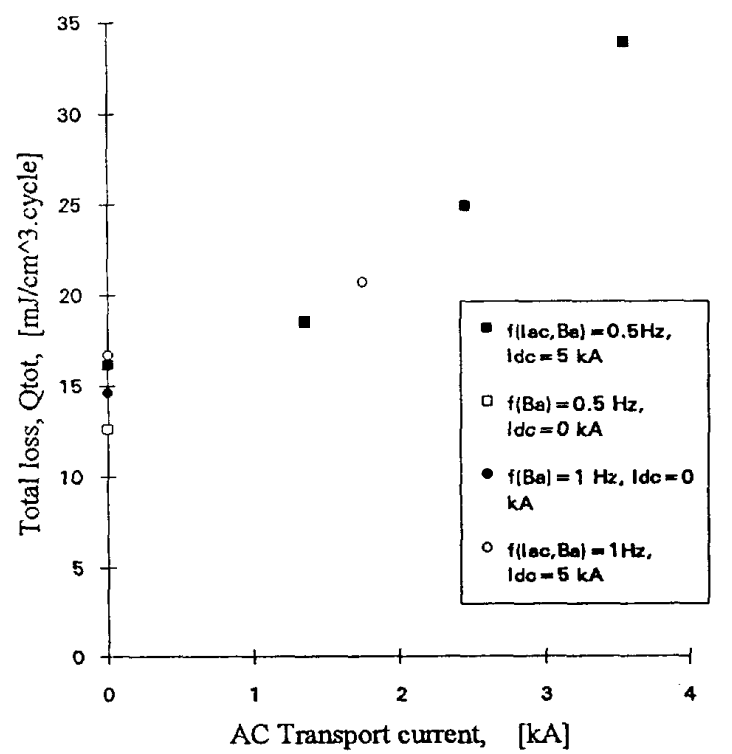

Fig. $2 \mathrm{Q}_{\text {tot }}=\mathrm{f( \textrm {I } _ { \mathrm { Tac } }}$ ), with transverse ac field $\mathrm{B}_{\mathrm{a}}=200 \mathrm{mT}, \mathrm{I}_{\mathrm{Tdc}}=5 \mathrm{kA}, \mathrm{B}_{\mathrm{dc}}=2 \mathrm{~T}$.

implies that under these circumstances the major part of the dissipation occurs in the superconducting material and the coupling loss can be disregarded [6]. The main part of the loss consists of hysteresis so the cubic behaviour found for low amplitudes of the ac transport current is explained by the cubic behaviour of the hysteresis loss at low currents producing ac fields smaller than the penetration field Bp and the cubic behaviour of transport current loss at low values of $\mathrm{I}_{\mathrm{T}} / \mathrm{I}_{\mathrm{c}}$. In the case the amplitude of the transport current exceeds $7 \mathrm{kA}$, the increase of loss as a function of transport current will become less than cubic because the produced self fields exceed $\mathrm{B}_{\mathrm{p}}$.

The results presented in Figure 2 give an impression of the losses caused by an ac transport current in combination with an external applied ac field. The dc transverse background field is $2 \mathrm{~T}$ and the de transport current $5 \mathrm{kA}$. The unidirectional ac transport current is synchronised with the sinusoidal transverse ac field with an amplitude of 200 $\mathrm{mT}$ and a frequency of 0.5 and $1 \mathrm{~Hz}$. Within the measured range it seems possible to add both loss components with a different origin, ac transport current and applied ac field.

\section{SIMULTANEOUSLY APPLIED TRANSVERSE AND LONGITUDINAL AC FIELDS.}

\section{A. Transverse and longitudinal ac fields ( no $I_{7}$ )}

In the TF coils of a tokamak the pulse field can have any orientation with respect to the filament axis. The influence

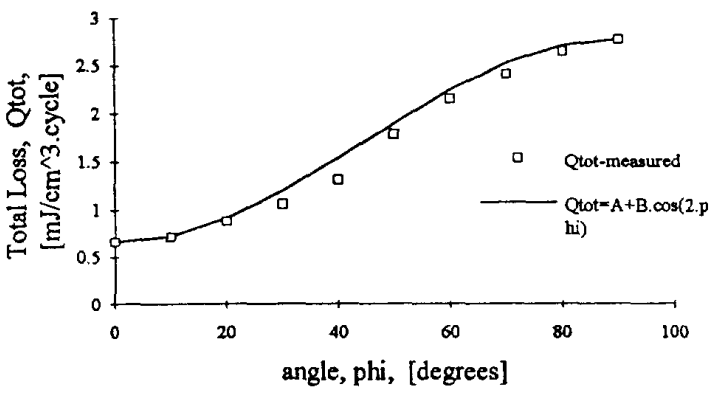

Fig. 3. Influence of the angle of the applied $2 c$ field with respect to the axis of the \#T1 cable $\left(f=8.1 \mathrm{~Hz}, \mathrm{~B}_{\mathrm{dc}}=2 \mathrm{~T}\right)$.

of the angle $\varphi$ between the applied field and the cable axis has been investigated experimentally.

The influence of the angle on the applied ac field with respect to the cable, can be studied using both field coils simultaneously. When the total amplitude is kept constant and by varying the ratio between both amplitudes any angle can be selected. The background field is $2 \mathrm{~T}$ and the amplitude of the sine wave ac field is $100 \mathrm{mT}$. An angle of 0 degrees means a pure longitudinal field and 90 degrees means a pure transverse field with respect to the cable axis (not to the strands).

The result of the experiment with sample \#T1 is shown in Figure 3. The line is a fit of the total loss to the expression $A+B \cdot \cos 2 \varphi$, where $A+B$ represents the measured loss in longitudinal field and A-B the loss in the purely transverse field. There is a good agreement between the measured points and the fitted curve.

\section{B. Transverse, longitudinal ac fields and transport current.}

With the twisted cable \#T1, an experiment is carried out in which the effect of simultaneously applied transverse and longitudinal fields are studied for different amplitudes $(\mathrm{Ba}=50,100$ and $150 \mathrm{mT})$ and frequencies of the ac field with a background field $B_{d c}=2 T$ and with a dc transport current of 0 and $10 \mathrm{kA}$. Some results of these experiments are shown in Figure 4 [2].

In order to distinguish the difference between separate and simultaneous action of both ac fields, both separately measured ac field contributions are added with the use of linear regression and compared to the measured results of the combined case. The result of this procedure is shown in Figure 5.

It is demonstrated in all cases that the loss due to the combined field exceeds the value of the added separate loss within about $0-10 \%$ in the frequency range up to $25 \mathrm{~Hz}$ and field sweep rate up to $\mathrm{dB} / \mathrm{dt}=24 \mathrm{~T} / \mathrm{s}$. Simultaneously applied transverse and longitudinal fields can result in a loss that exceeds the added contributions obtained with the 


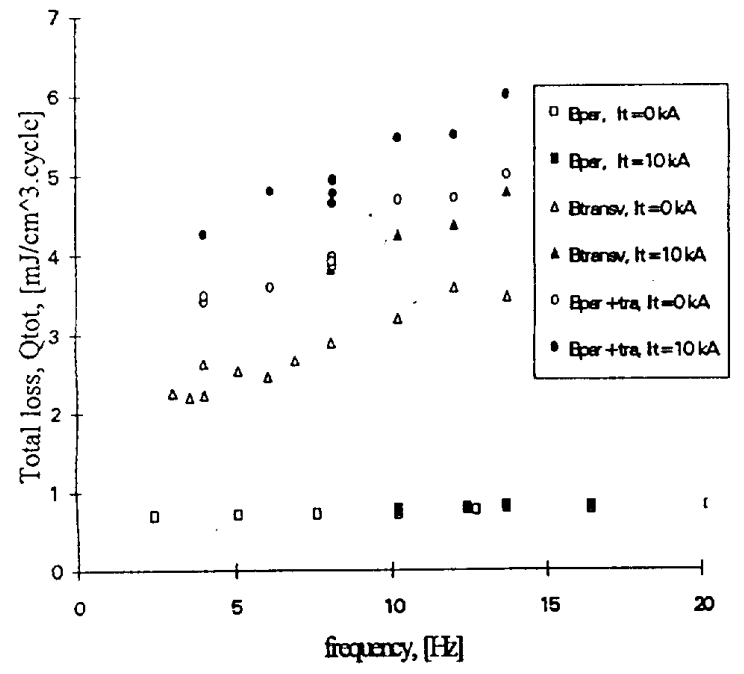

Fig. 4 Simultaneously applied transverse and longitudinal fields for the \#T1, with $B_{2}=100 \mathrm{mT}$ and $B_{d c}=2 T$.

separately applied ac fields. In the case of the presence of a dc transport current the same effect occurs but the effect is less than without current.

\section{Conclusions.}

By using the new test set-up it is possible to measure ac loss caused by transverse or longitudinal applied ac fields, acting separately or simultaneously, in combination with dc and ac transport currents and in a background field of $2 \mathrm{~T}$.

The penetration fields, hysteresis and coupling loss are determined for transverse and longitudinal ac fields at different background fields without transport current.

It was found that the hysteresis loss in a longitudinal field, in the case the field exceeds the penetration field, is 10 - 20 times smaller than in a transverse field depending on the type of strand.

A comparison of the produced coupling loss for different types of cables, show that in a transverse ac field the braided cable has an approximately 1.5 times higher loss than the twisted cables.

In parallel ac fields this relation is opposite as both twisted cables show 1.8 and 1.4 times respectively higher coupling losses than the braided cable.

The relations between the loss and the ramp rate of the ac transport current, measured with different amplitudes, proves that the main part of the loss consists of self field loss. In the range investigated it is allowed to simply add the

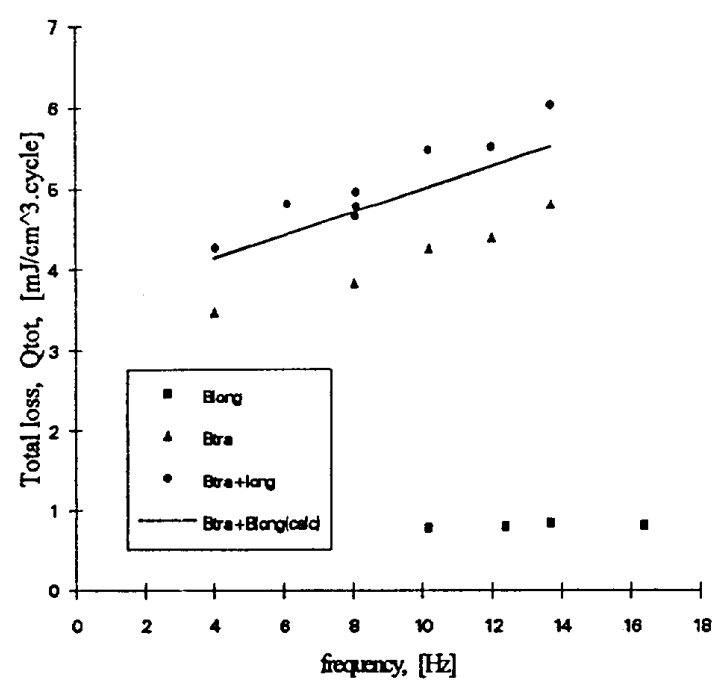

Fig. 5. Total loss for separate and combined transverse and longitudinal ac fields, with transport current $I_{T d c}=10 \mathrm{kA}$ and $B_{d c}=2 T\left(B_{a}=100 \mathrm{mT}\right)$.

loss component of the self field and the loss caused by the applied transverse ac field.

Application of transverse and longitudinal ac fields simultaneously and a background field, can result in a total loss that exceeds (within $10 \%$ ) the sum of the contributions caused by the separate applied ac fields. In the case a dc transport current is present, the same effect occurs although the effect is less than without current. The measured total loss is always above the sum of the loss components.

\section{REFERENCES.}

[1] G.B.J. Mulder, et al 'A new test set up to measure the AC losses of the conductors for NET, IEEE Trans on Magn., MAG-27, vol 2, 1991, p. 2190.

[2] A. Nijhuis and H.H.J. ten Kate, 'AC loeses with transport current in NET prototype subcables.' Final Report UT-NET 93/3, Contract no. NET/91277, University Twente, May 1993.

[3] A. Nijhuis and H.H.J. ten Kate, 'Study of the effect of transverse and longitudinal fields on the ac loss of NET prototype conductors', (to be publishod).

[4] A. Nijhuis and H.H.J. ten Kate, Interstrand coupling loss in NET prototype cabled onductors carrying a de transport current', paper will be presented at EUCAS'93, October 1993, Gottingen, Germany.

[5] P. Bruzzone ef al.,'AC Losses for the prototype cable in conduit conductors for NET, IEEE Trans on Magn., vol.28, no.1, 1992, pp.194-197.

[6] J.L. de Reuver, 'AC Losses in current-carrying superconductors', thesis, University of Twente, Enschede, December 1984. 